\title{
PARAMETRIC MODELS FOR ESTIMATING WIND TURBINE FATIGUE LOADS FOR DESIGN
}

\author{
Lance Manuel ${ }^{\mathrm{l}}$ \\ Paul S. Veers ${ }^{2}$ \\ Steven R. Winterstein ${ }^{3}$ \\ ${ }^{1}$ Department of Civil Engineering, University of Texas at Austin, Austin, TX 78712 \\ ${ }^{2}$ Sandia National Laboratories, Wind Energy Technology Department, Albuquerque, NM 87185-0708 \\ ${ }^{3}$ Department of Civil and Environmental Engineering, Stanford University, Stanford, CA 94305-4020
}

\begin{abstract}
International standards for wind turbine certification depend on finding long-term fatigue load distributions that are conservative with respect to the state of knowledge for a given system. Statistical models of loads for fatigue application are described and demonstrated using flap and edge blade-bending data from a commercial turbine in complex terrain. Distributions of rainflow-counted range data for each ten-minute segment are characterized by parameters related to their first three statistical moments (mean, coefficient of variation, and skewness). Quadratic Weibull distribution functions based on these three moments are shown to match the measured load distributions if the non-damaging low-amplitude ranges are first eliminated. The moments are mapped to the wind conditions with a two-dimensional regression over ten-minute average wind speed and turbulence intensity. With this mapping, the short-term distribution of ranges is known for any combination of average wind speed and turbulence intensity. The longterm distribution of ranges is determined by integrating over the annual distribution of input conditions. First, we study long-term loads derived by integration over wind speed distribution alone, using standard-specified turbulence levels. Next, we perform this integration over both wind speed and turbulence distribution for the example site. Results are compared between standarddriven and site-driven load estimates. Finally, using statistics based on the regression of the statistical moments over the input conditions, the uncertainty (due to the limited data set) in the long-term load distribution is represented by $95 \%$ confidence bounds on predicted loads.
\end{abstract}

\footnotetext{
This paper is declared a work of the U.S. Government and is not subject to copyright protection in the United States. Sandia is a multiprogram laboratory operated by Sandia Corporation, a Lockheed Martin company, for the U.S. Department of Energy under contract DE-AC04-94AL85000.
}

\section{INTRODUCTION}

Design constraints for wind turbine structures fall into either extreme load or fatigue categories. In the case of extreme load design drivers, the load estimation problem is limited to finding a single maximum load level against which to assess the structural strength. For design against fatigue, however, loads must be defined over all input conditions and then summed over the distribution of input conditions weighted by the relative frequency of occurrence. While this might seem to be a more daunting task, it is in many ways quite similar to the extreme load problem, as can be seen by comparing with Fitzwater and Winterstein ${ }^{1}$. In both cases, the loads must be determined as functions of wind speed (or other climatic conditions).

Parametric models define the response, statistically, with respect to input conditions. Such models fit analytical distribution functions to the measured or simulated data. The parameters of these distribution functions can be useful in defining the response/loads as a function of the input conditions. The end result, then, is a full statistical definition of the loads over all input conditions.

In the most prevalent alternative to parametric modeling, an empirical distribution of loads (i.e., a histogram describing frequency of occurrence of the modeled response quantity) is used to define the turbine response at the conditions of the measurement or simulation. When using simulations, a ten-minute time series is generated at specified environmental conditions using an aeroelastic analysis code. The time series is rainflow-counted and the number of ranges in specified intervals is summarized in histograms. The histograms serve as empirical distributions that are taken to be representative of the response of the turbine at those particular conditions. The full lifetime distribution is then obtained by summing the distributions after weighting by the frequency of occurrence of the wind speed associated with each simulated data segment included in a histogram interval. In the case of measured data, a similar 


\section{DISCLAIMER}

This report was prepared as an account of work sponsored by an agency of the United States Government. Neither the United States Government nor any agency thereof, nor any of their employees, make any warranty, express or implied, or assumes any legal liability or responsibility for the accuracy, completeness, or usefulness of any information, apparatus, product, or process disclosed, or represents that its use would not infringe privately owned rights. Reference herein to any specific commercial product, process, or service by trade name, trademark, manufacturer, or otherwise does not necessarily constitute or imply its endorsement, recommendation, or favoring by the United States Government or any agency thereof. The views and opinions of authors expressed herein do not necessarily state or reflect those of the United States Government or any agency thereof. 


\section{DISCLAIMER}

Portions of this document may be illegible in electronic image products. Images are produced from the best available original document. 
approach has been described by McCoy et al. ${ }^{2}$ but with an innovative weighting scheme to account for the irregular input conditions of field measurements.

The empirical approach uses only the measured or simulated data without any fitting of distributions or extrapolation to higher values that would be seen if more data were obtained. One of the disadvantages of using a purely empirical approach is, therefore, that the loading distribution may not be representative. Perhaps a subtler shortcoming is that the uncertainty in the loads is almost impossible to characterize.

With regard to uncertainties in loads and how they might be dealt with in design, one might expect that these uncertainties could be covered by the use of standard specifications of characteristic loads (derived from a specified high turbulence level) and safety factors. However, current standard load definitions use safety factors that do not depend on the relative uncertainty in the load estimates. Either the margins are larger than they need to be when load estimates are reasonably well established (i.e., exhibit low uncertainty), or they fail to cover the uncertainty when load estimates are based on limited data (i.e., large uncertainty cases).

Parametric load distribution models offer significant advantages over empirical models; they provide a means to (1) extrapolate to higher, less frequent load levels, (2) map the response to the input conditions, and (3) calculate load uncertainty. For example, Ronold et al. ${ }^{3}$ have published a complete analysis of the uncertainty in a wind turbine blade fatigue life calculation. They used a parametric definition of the fatigue loads, matching the first three moments of the wind turbine cyclic loading distribution to a quadratic (transformed by a squaring operation) Weibull distribution.

Veers and Winterstein ${ }^{4}$ described a parametric approach, quite similar to that employed by Ronold et al. $^{3}$, that can be used with either simulations or measurements, and have shown how it may be used in an uncertainty evaluation. Although Reference 4 describes how to use the statistical model to estimate the complete load spectrum, it does not indicate how these models compare with the design standards ${ }^{5}$. It is critical that the load distributions generated by any statistical methodology be adaptable for use in existing design standards. Moreover, it is arguably even more important that the load model provide insight into how the design standards might be improved in future revisions. The standards should require an accurate reflection of the load distribution with sufficient conservatism to cover the uncertainties caused by the limited duration of the sample, whether Based oh simulation or field measurements. Only then can design margins be trimmed to the point of least cost while still maintaining sufficient margins to keep reliability levels high.

The approach to load modeling is not uniform across the wind community by any measure. This lack of commonality in approach was reflected in the working group that produced IEC's Mechanical Load Measurement Technical Specification ${ }^{6}$. No consensus could be obtained on how to use measured loads to either create or substantiate a fatigue load spectrum at the conditions specified in the Safety Standard ${ }^{5}$. All that is offered are several examples of differing approaches in an annex of the specifications ${ }^{6}$.

Here, we present a methodology for using measured or simulated loads to produce a long-term fatigue-load spectrum at specified environmental conditions and at desired confidence levels. To illustrate, example measurements of the two blade-root moments (flap and edge) from a commercial turbine in complex terrain are used. The ten-minute distributions of rainflow ranges are modeled by a quadratic Weibull distribution function based on three statistical moments of the ranges (mean, coefficient of variation, and skewness). The moments are mapped to the wind conditions by a two-dimensional regression over ten-minute average wind speed and turbulence intensity. Thus, the "shortterm" distribution of ranges may be predicted for any combination of average wind speed and turbulence intensity. The "long-term" distribution of ranges is, then, easily obtained by integrating over the annual distribution of input conditions. Results are compared between standard-driven and site-driven load estimates. Finally, using statistics based on the regression of the statistical moments over the input conditions, the uncertainty (due to the limited data set) in the long-term load distribution is represented by $95 \%$ confidence bounds on predicted loads.

\section{IEC LOAD CASES}

The loads specified by IEC 61400-1 Wind Turbine Generator Safety Requirements for design must be defined for a specified combination of mean wind speed and turbulence intensity known as the Normal Turbulence Model ${ }^{5}$. The standard provides an equation for the standard deviation of the ten-minute wind speed, $\sigma_{1}$, that depends on the hub-height wind speed and two parameters, $I_{15}$ and $a$.

$$
\sigma_{1}=I_{15}\left(15 \mathrm{~m} / \mathrm{s}+a V_{h u b}\right) /(a+1)
$$


Equation 1 is based on wind speed standard deviation data gathered from around the world and aggregated into a common data set. The equation was created to be "broadly representative of sites with reasonable international marketing interest," ${ }^{8}$ and does not represent any single site. $\sigma_{1}$ is intended to represent a characteristic value of wind-speed standard deviation. Certification guidelines are provided for high $(A)$ and moderate (B) turbulence sites. $I_{15}$ defines the characteristic value of the turbulence intensity at an average wind speed of $15 \mathrm{~m} / \mathrm{s}$, and $a$ is a slope parameter when $\sigma_{1}$ is plotted versus hub-height wind speed. The values of these parameters for each category are shown in Table 1.

\begin{tabular}{|c|c|c|}
\hline CATEGORY & $\begin{array}{c}\text { A } \\
(\mathrm{HIGH})\end{array}$ & $\begin{array}{c}\text { B } \\
\text { (MODERATE) }\end{array}$ \\
\hline$I_{15}$ & 0.18 & 0.16 \\
\hline$a$ & 2 & 3 \\
\hline
\end{tabular}

Table 1: Parameters for IEC turbulence categories.

The Category B moderate turbulence specification is intended to roughly envelope (i.e., be higher than) the mean plus one sigma level of turbulence for all the collected data above $15 \mathrm{~m} / \mathrm{s}$. Similarly, Category A envelopes all collected values of turbulence intensity (with the exception of one southern California site) for mean wind speeds above $15 \mathrm{~m} / \mathrm{s}$ and is above the overall mean plus two sigma level in high winds $^{8}$. Clearly, the IEC Normal Turbulence Model is intended to be conservative for all but the most turbulent sites.

It is a relatively straightforward matter to create a loading distribution that meets the standard criteria when using an aeroelastic simulation code. Input winds can be generated for any combination of wind speed and turbulence intensity. Representative loadings can, in theory, be generated by simulating repeatedly until sufficient data are produced to drive the uncertainty to an arbitrarily small level. Practically, however, it would be beneficial to generate a loading distribution with small, or at least known, uncertainty from a smaller data set. This is where the parametric approach provides significant value. By means of regression of load statistics (e.g., moments) over the entire range of wind speeds and turbulence levels, the uncertainty in the values of the parameters defining the short-term distributions at any specified turbulence condition can be estimated.

In the case of measured loads, it may be simply impossible to gather data at the specified turbulence conditions because of the limitations of the test site. In that case, the parametric approach provides a method to interpolate to a specified turbulence level using all of the data collected (thus adding to the confidence of the interpolation), or to extrapolate beyond the limits of the measurements. In either case, the parametric approach simplifies the generation of fatigue loads to Standard specifications.

\section{EXAMPLE DATA SET}

An example data set taken from the copious measurements of the MOUNTURB program ${ }^{7}$ is used to illustrate the parametric modeling process. The data are comprised of 101 ten-minute samples of rainflowcounted flap-wise and edge-wise bending-moment ranges at the blade root. The test turbine is a WINCON $110 \mathrm{XT}$, a $110 \mathrm{~kW}$ stall-regulated machine operated by CRES (the Centre for Renewable Energy Systems, Pikermi, Greece) at their Lavrio test site. The terrain is characterized as complex.

The original time series of the loads and winds were not available for further analysis; thus, only the rainflowcounted ranges were employed. The number of cycle counts was tallied in 50 bins ranging from zero to the maximum range in each sample. A single ten-minute sample is categorized by the mean wind speed and the raw turbulence intensity at hub height. The average wind speeds are limited to the range between 15 and 19 $\mathrm{m} / \mathrm{s}$ and thus reflect response in high wind operation. Turbulence intensities cover a wide range of operating conditions as can be seen in Figure 1. The measured loads are summarized by frequency of occurrence in Figure 2a for flap moment ranges and in Figure $2 c$ for edge moment. Plots showing exceedance counts for specified flap and edge loads are shown in Figures $2 b$ and $2 \mathrm{~d}$, respectively.

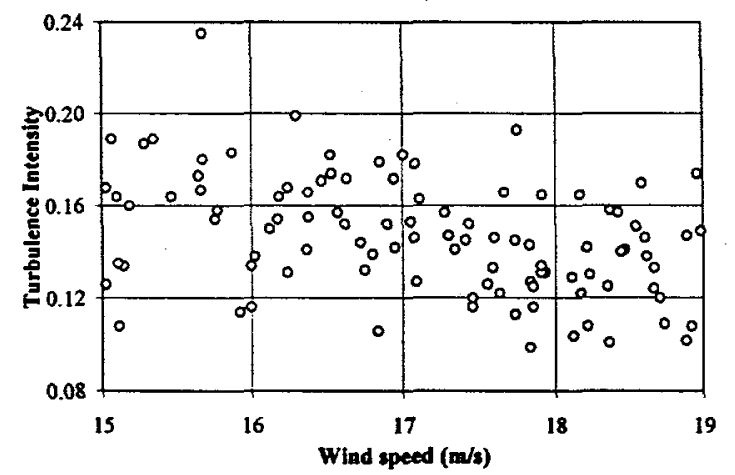

Figure 1 Wind speed and turbulence intensity values for the 101 10-minute data samples. 


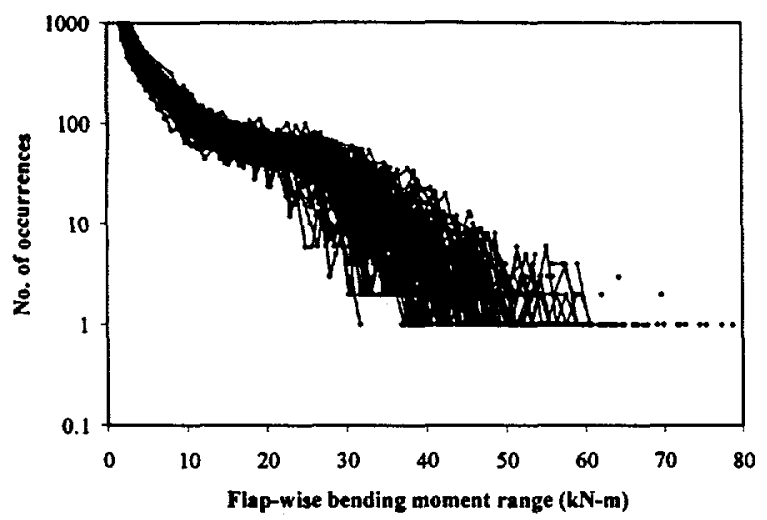

Figure 2a Histogram of flap-wise bending moment ranges for 10110 -minute data sets.

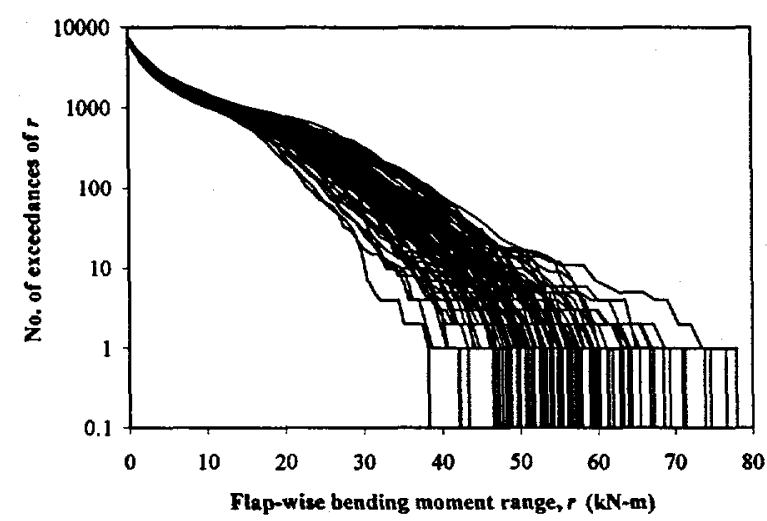

Figure $2 b$ Cumulative counts of flap-wise bending moment ranges for 101 10-minute data sets.

\section{SHORT-TERM ANALYSIS}

The FITS $^{9}$ software for fitting probability distribution models to empirical data was used to analyze each tenminute sample. FITS calculates the central moments of the data and estimates the best fit distribution model to match a user-specified set of moments (e.g., the user can request a distribution model fit based on two moments or one based on three moments). FITS is, therefore, a tool for examining the fit of a probability model to the short-term response, conditional on wind speed and turbulence level.

For purposes of the present discussion, the first three moments $\mu_{i},(i=1,2,3)$ of the rainflow-range amplitudes, $r$, are defined here as:

$$
\begin{aligned}
& \mu_{1}=E[r]=\bar{r}, \\
& \mu_{2}=\frac{\sigma_{r}}{\bar{r}} ; \sigma_{r}{ }^{2}=E\left[(r-\bar{r})^{2}\right],
\end{aligned}
$$

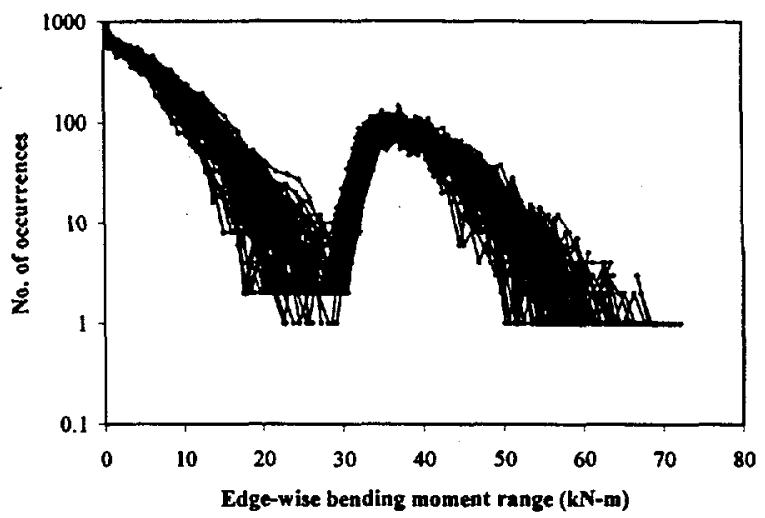

Figure 2c Histogram of edge-wise bending moment ranges for 101 10-minute data sets.

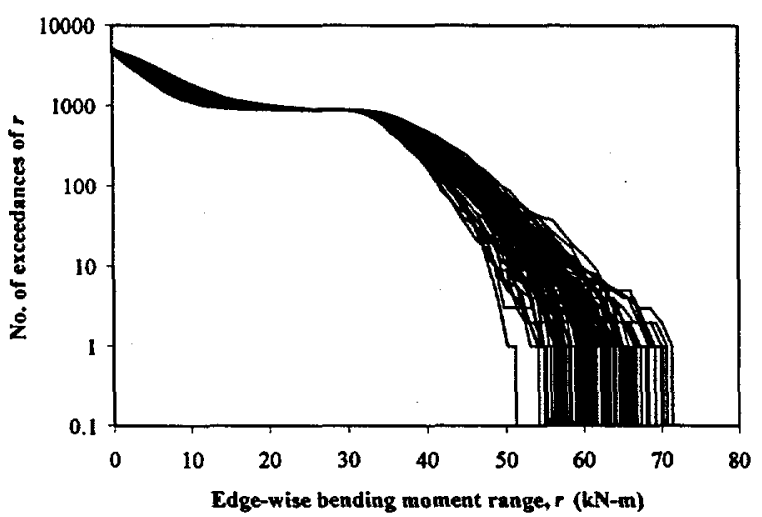

Figure 2d Cumulative counts of flap-wise bending moment ranges for 101 10-minute data sets.

$\mu_{3}=\frac{E\left[(r-\bar{r})^{3}\right]}{\sigma_{r}{ }^{3}}$

where $\mathrm{E}[\bullet]$ is the expectation (or averaging) operator. The first moment is the mean or average range, a measure of central tendency. The second moment is the Coefficient of Variation (COV), which is the standard deviation divided by the mean, a measure of the dispersion or spread in the distribution. The third moment is the coefficient of skewness, which provides information on the tail behavior of the distribution. Load range data are often well fit by a Weibull distribution, a slight distortion of the Weibull distribution is used to exactly match the first three statistical moments ${ }^{10}$.

To illustrate the fit of the quadratic Weibull distribution to a ten-minutes sample, one of the 101 samples shown in Figure 2 is studied. This data sample is taken from the middle of the measured wind conditions; $V=17 \mathrm{~m} / \mathrm{s}$ and $I=0.18$. The data are plotted on a Weibull scale for the flap loads in Figure 3 and for the edge loads in Figure 4. The vertical scale is transformed from the 


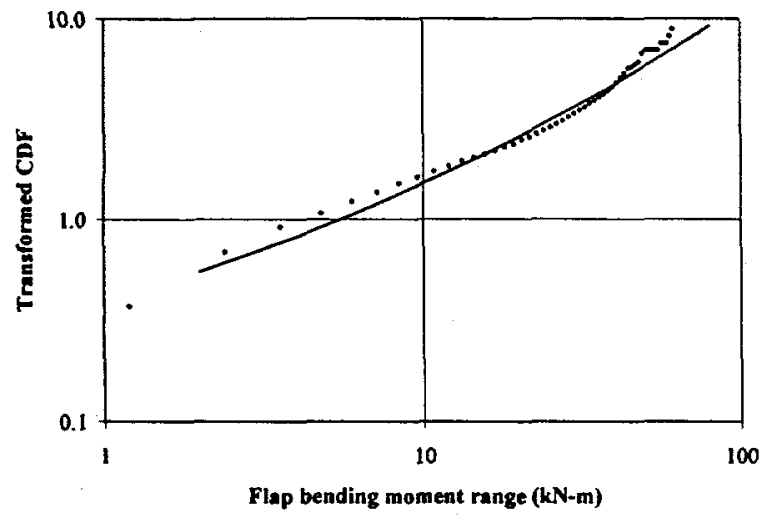

(a) Weibull scale plot.

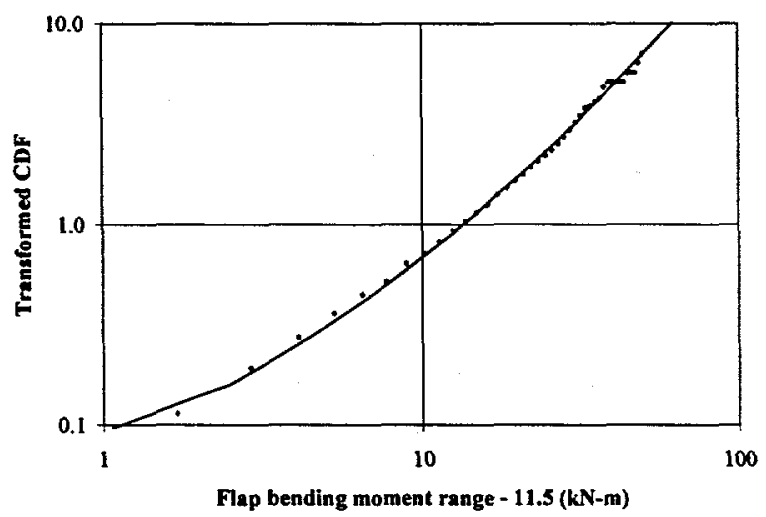

(b) Weibull scale plot (truncation at $11.5 \mathrm{kN}-\mathrm{m}$ ).

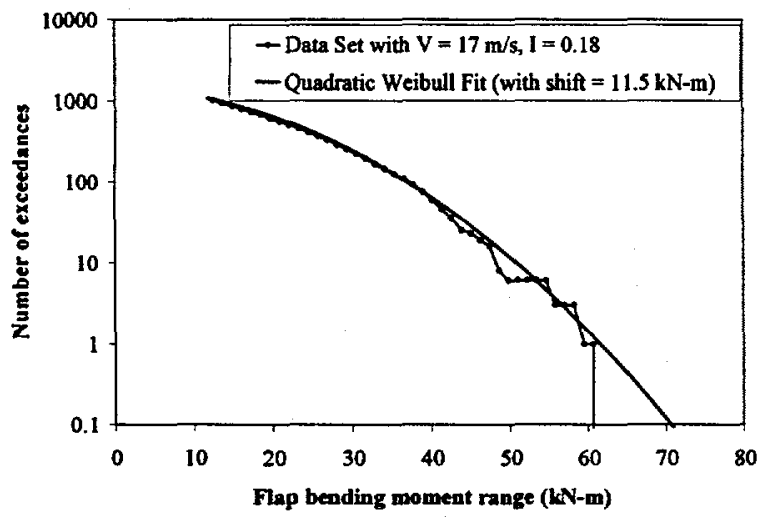

(c) Exceedance plot format (truncation at $11.5 \mathrm{kN}-\mathrm{m}$ ).

Figure 3 Quadratic Weibull model fits to data on flapbending moment ranges $(V=17.0 \mathrm{~m} / \mathrm{s}, I=$ 0.18 ).

Cumulative Distribution Function (CDF) as $(-\ln (1-$ $\mathrm{CDF}$ )) so that a Weibull distribution will be a straight line on a $\log -\log$ plot. (Recall that the CDF is the complement of the traditional exceedance diagram; exceedance $=1-\mathrm{CDF}$.)

Figures $3 a$ and $4 a$ show attempts to fit the entire flap and edge data with quadratic Weibull models. As seen

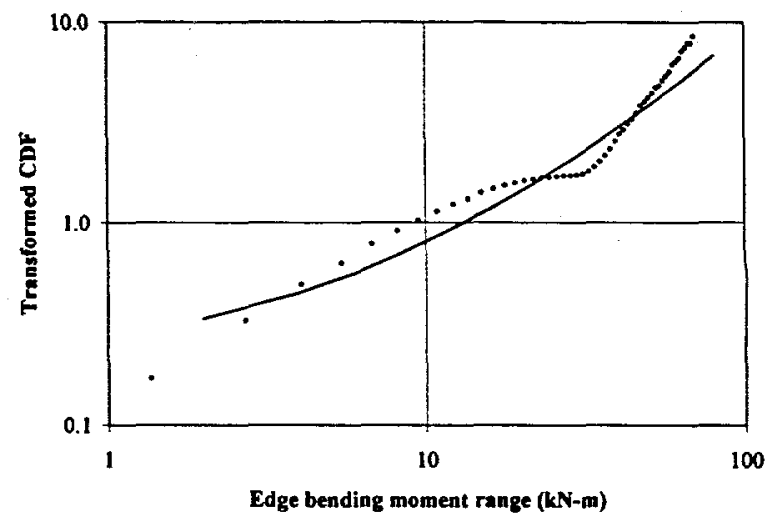

(a) Weibull scale plot.

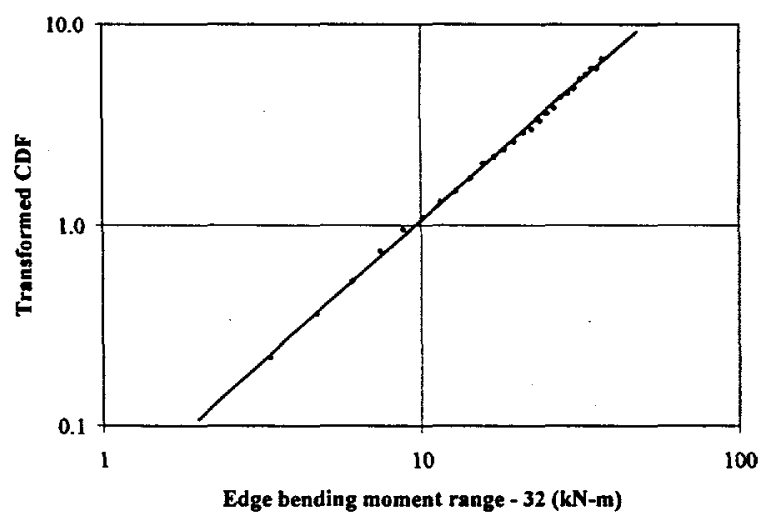

(b) Weibull scale plot (truncation at $32.0 \mathrm{kN}-\mathrm{m}$ )

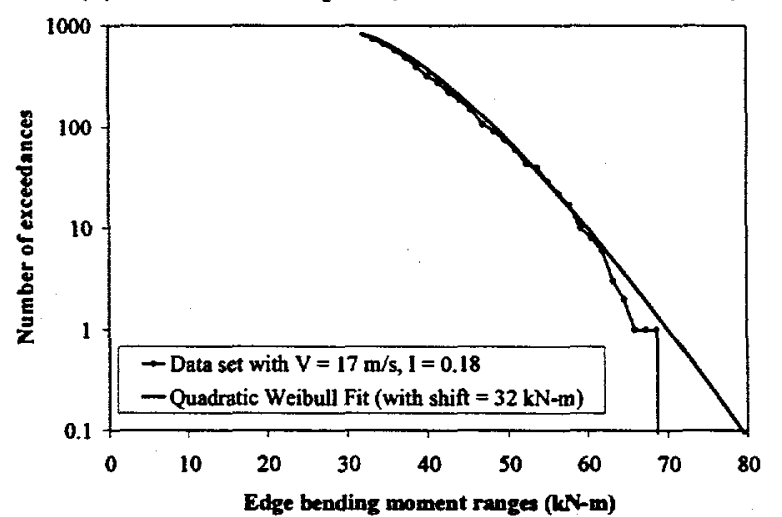

(c) Exceedance plot format (truncation at $32.0 \mathrm{kN}-\mathrm{m}$ ).

Figure 4 Quadratic Weibull model fits to data on edgebending moment ranges $(V=17.0 \mathrm{~m} / \mathrm{s}, I=$ 0.18).

in Figure $3 a$ and especially Figure $4 a$, the data have a kinked appearance which the smooth probability distribution, in spite of the quadratic distortion, can not match. Closer examination of the data reveals that the kink is due to a very large number of cycles at relatively low amplitudes. 
The proliferation of small amplitude cycles seen in Figure 2 produces a distribution difficult to duplicate with a simple analytical form, but these small cycles produce relatively little damage. By truncating the loads at a threshold, the kink in the data can be eliminated without significantly reducing the damage. In the edge case, there are obviously a great number of cycles of smaller amplitude than the dominant gravity load at about $32 \mathrm{kN}-\mathrm{m}$. The flap loads have a less distinctive kink at around 10-13 kN-m (11.5 kN-m was used as the filtering threshold). Figures $3 \mathrm{~b}$ and $4 \mathrm{~b}$ are similar to Figures $3 a$ and $4 a$ but include only a subset of the data and can be thought of as applying a "shift" to all loads that effectively discards the smallest cycles. Clearly, the fits of the quadratic Weibull are improved dramatically. Thus, the short-term data are well modeled by a quadratic Weibull distribution that preserves the first three central moments of the truncated rainflow ranges.

Figures $3 c$ and $4 c$ show the same data as do Figures $3 b$ and $4 \mathrm{~b}$ but with the axes in the more common exceedance plot format. These plots are included to

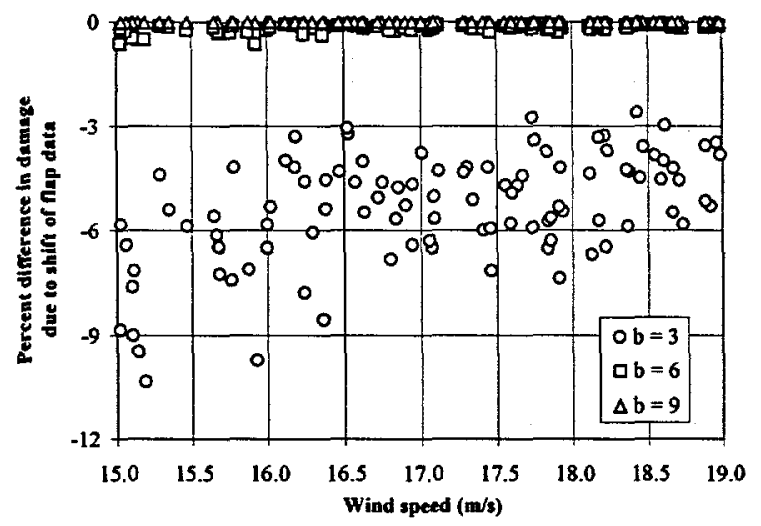

(a) Flap-wise bending

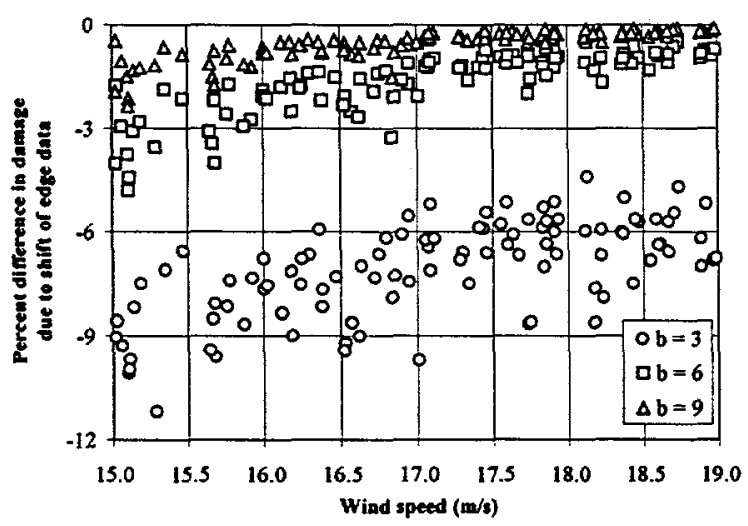

(b) Edge-wise bending

Figure 5 Effect on damage estimation of shift in blade bending moment range data. reorient the reader back to the original summaries of the data shown in Figure 2. They also serve to illustrate how the analytical distribution functions may be used to extrapolate to less frequent, higher amplitude loads.

The low amplitude cycles (that make distribution fits difficult as described in the preceding) can only be discarded if they produce an insignificant amount of damage. The damage unaccounted for due to the truncation of rainflow range data at $11.5 \mathrm{kN}-\mathrm{m}$ for the flap loads is represented in Figure $5 \mathrm{a}$, and due to a truncation at $32 \mathrm{kN}-\mathrm{m}$ for the edge loads in Figure $5 \mathrm{~b}$. All 101 ten-minute data segments are represented in Figures $5 \mathrm{a}$ and $5 \mathrm{~b}$. Lost damage is plotted for three fatigue exponents, $b$, representing typical values of wind turbine materials ranging from $b=3$ in welded steel up to $b=9$, more characteristic of fiberglass composites. In no case does the truncation remove more that $12 \%$ of the damage, and that only when $b=3$. In almost all cases for the higher $b$ values, the lost damage is less than $3 \%$. In both flap and edge bending cases, over $80 \%$ of the rainflow-counted ranges are removed by truncating the data sets. Our findings that discarding so much of the data does not lead to grossly unconservative estimates of damage is not unexpected since it has long been known that eliminating most of the small amplitude cycles has a negligible effect on damage ${ }^{11}$.

\section{REGRESSION ANALYSIS}

Because a good match can be obtained to the short-term distribution of rainflow ranges given the first three moments and a fixed data truncation, it is sufficient to know the moments over all the operating conditions in order to fully define the turbine fatigue loading. Regression of the moments over ten-minute average wind speed and turbulence intensity can achieve the desired result and assist in understanding the loading dependence on and sensitivity to both turbulence and wind speed. Results from a regression analysis can also provide information on the uncertainty of the loads.

The moments presented in the following figures, $\mu_{2}$ and $\mu_{3}$ describe the COV and skewness, respectively, of the shifted range $r^{\prime}=r-r_{1}$. More precisely, by eliminating all ranges below the truncation level $r_{i}$, we obtain the shifted values $r^{\prime}=r-r_{\text {, }}$ of the remaining ranges and consider models based on statistics of $r_{i \text {. }}$. This is done to conform with the quadratic Weibull model, which generally assigns probability to all outcomes $r^{\prime}>0$. For example, the second moment is

$$
\mu_{2}=\frac{\sigma_{r}}{\bar{r}-r_{t}}
$$




\begin{tabular}{|c|c|c|c|}
\hline & COEFFICIENT & $\underline{\text { FLAP }}$ & EDGE \\
\hline \multirow{3}{*}{$\sum_{\Sigma}^{\mathbb{E}}$} & $a_{l}$ & 21.49 & 40.02 \\
\hline & $b_{1}$ & 0.808 & 0.359 \\
\hline & $c_{l}$ & 0.202 & 0.039 \\
\hline \multirow{3}{*}{8} & $\overline{a_{2}}$ & 0.722 & 0.635 \\
\hline & $b_{2}$ & 0.031 & -0.573 \\
\hline & $c_{2}$ & 0.080 & 0.063 \\
\hline \multirow{3}{*}{ 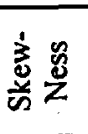 } & $a_{3}$ & 0.963 & 0.980 \\
\hline & $b_{3}$ & -1.260 & -0.468 \\
\hline & $\frac{c_{3}}{c_{3}}$ & 0.033 & 0.132 \\
\hline \multicolumn{4}{|c|}{ Reference values: $V_{\text {ref }}=17.1 \mathrm{~m} / \mathrm{s} ; I_{\text {ref }}=0.145$} \\
\hline
\end{tabular}

Table 2: Coefficients from regression analysis.

Because these definitions of $\mu_{2}$ and $\mu_{3}$ are dimensionless, they are insensitive to the choice of truncation level. For example, if the loads are exponentially distributed, $\mu_{2}=1$ and $\mu_{3}=2$ regardless of the truncation level. The mean value, $\mu_{1}$, presented is the same as $\bar{r}$, the mean with respect to zero of the ranges retained after eliminating the small-amplitude cycles.

As in Reference 4 , the first three moments $\left(\mu_{i}, i=1-3\right)$ are fitted to a power law function of wind speed, $V$, and turbulence intensity, $I$.

$\mu_{i}=a_{i}\left(\frac{V}{V_{\text {ref }}}\right)^{b_{i}}\left(\frac{I}{I_{\text {ref }}}\right)^{c_{i}}$

The reference wind speed, $V_{\text {ref, }}$ and reference turbulence intensity, $I_{\text {ref }}$, are determined from the geometric mean values of the data ${ }^{4}$. For the Lavrio data set, $V_{\text {ref }}=17.1 \mathrm{~m} / \mathrm{s}$ and $I_{\text {ref }}=0.145$. The calculated regression coefficients are shown in Table 2 .

The regression results for the flap bending moments are shown in Figure 6 and for the edge bending moments in Figure 7. Mean, COV, and skewness are plotted in the parts (a), (b), and (c), respectively, of the figures. In all cases, the regression line uses the reference value for turbulence intensity. The circles correspond to the measured data from the 101 samples. The solid symbols (squares) show the regression prediction using the measured wind speed and turbulence intensity for each ten-minute data set. A large spread in solid symbols about the regression line indicates a sizeable dependence on turbulence level (e.g., Figure 6a's mean flap range) while a small variation in the solid symbols indicates that the turbulence has little effect on that particular moment (e.g., Figure 6c's flap skewness). This sensitivity can also be inferred from the magnitude of the $c_{i}$ coefficients in Table 2. The smaller the value

\begin{tabular}{||l|c|c|}
\hline \hline PARAMETER & FLAP & EDGE \\
\hline$R^{2}-$ Mean, $\mu_{1}$ & 0.51 & 0.76 \\
\hline$a_{1}(\sigma)$ & 0.108 & 0.050 \\
\hline$b_{1}(\sigma, t$ statistic $)$ & $(0.081,9.9)$ & $(0.020,17.5)$ \\
\hline$c_{l}(\sigma, t$ statistic $)$ & $(0.032,6.4)$ & $(0.008,4.9)$ \\
\hline$R^{2}-$ COV, $\mu_{2}$ & 0.05 & 0.43 \\
\hline$a_{2}(\sigma)$ & 0.003 & 0.003 \\
\hline$b_{2}(\sigma, t$ statistic $)$ & $(0.076,0.3)$ & $(0.080,7.1)$ \\
\hline$c_{2}(\sigma, t$ statistic $)$ & $(0.030,2.7)$ & $(0.031,2.0)$ \\
\hline$R^{2}-$ Skewness, $\mu_{3}$ & 0.17 & 0.05 \\
\hline$a_{3}(\sigma)$ & 0.018 & 0.016 \\
\hline$b_{3}(\sigma, t$ statistic $)$ & $(0.301,4.2)$ & $(0.266,1.8)$ \\
\hline$c_{3}(\sigma, t$ statistic) & $(0.117,2.8)$ & $(0.104,1.3)$ \\
\hline Avg. Cycle Rate & $1.75 \mathrm{~Hz}$ & $1.38 \mathrm{~Hz}$ \\
\hline
\end{tabular}

Table 3: Regression parameter summary.

of $c_{i}$, the less the importance of $I$ in the estimate of the $i^{\text {th }}$ moment.

Table 3 summarizes the regression uncertainties in terms of the widely used $R^{2}$ and $t$ statistics. A high $R^{2}$ value, approaching unity, indicates that a large percentage of the data variation is explained by the regression. In contrast, a low $R^{2}$ value suggests the presence of other influences, not included in the regression model, that induce the scatter in the data. Note that the goal here is not to predict the moment statistics in a single 10-minute history, but rather the long-run average of such 10-minute samples over the entire turbine lifetime.

The $t$ statistic, which is the estimated coefficient divided by the standard deviation of the estimate, indicates whether a particular coefficient is statistically significant. A $t$ value less than about two would indicate that the coefficient is not significantly different from zero at about the $95 \%$ confidence level. Since the leading coefficients, $a_{i}$, are estimates of the moments at the reference conditions, they are always significantly different from zero, and $t$ is not reported for them. However, the $t$ values of $b_{i}$ and $c_{i}$ indicate whether any functional variation with respect to wind speed or turbulence intensity, respectively, is supported by the data.

Examination of Tables 2 and 3 suggests that the mean load range is strongly related to both wind speed and turbulence, although the relation to turbulence has small exponents $(0.202$ and 0.039$)$. The only higher moment relationships that have high $t$ statistics are the edge $\mathrm{COV}$ relation to wind speed and the flap skewness relation to wind speed. The overall low exponents and $t$ statistics for the higher moments indicate that the distribution shapes are relatively constant over all input 


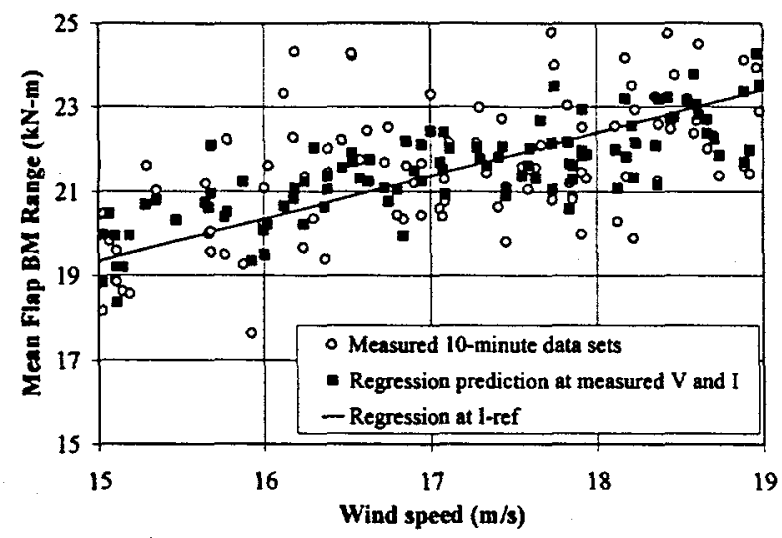

(a) Mean

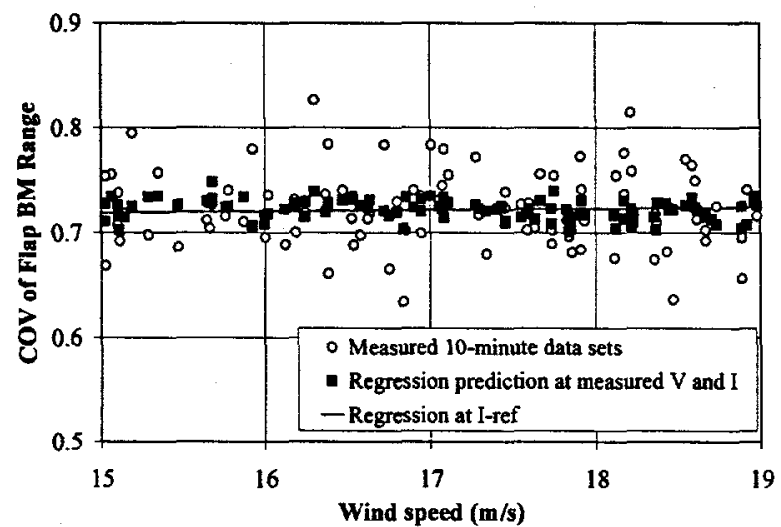

(b) Coefficient of variation (COV)

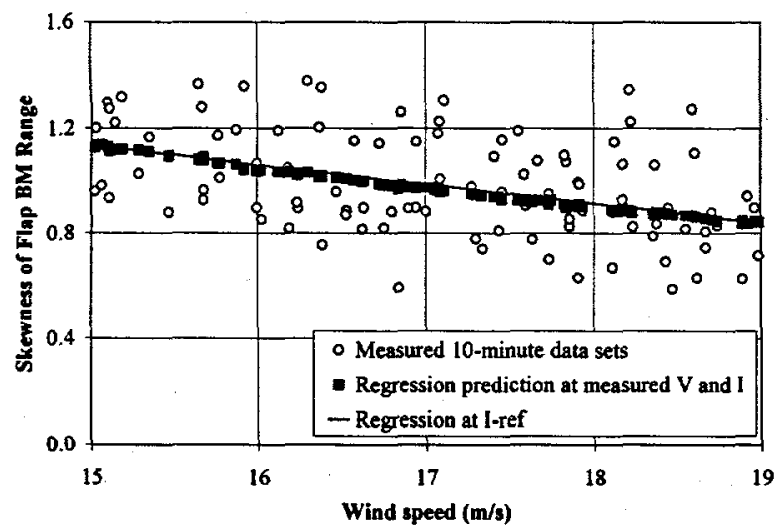

(c) Coefficient of skewness

Figure 6 Regression results for flap-wise bending moment range.

conditions. The variation seen in Figures 6 and 7 beyond that indicated by the solid symbols is sampleto-sample variation not indicative of a systematic relationship with the independent variables, $V$ and $I$. Part of this remaining variation will be irreducible, a natural outcome of random processes, but some could possibly be reduced with regression over better turbulence descriptors than the simple turbulence intensity.

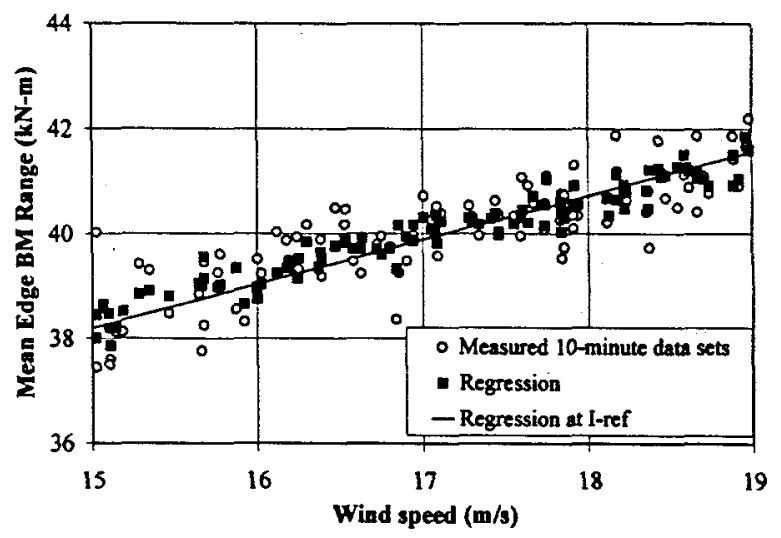

(a) Mean

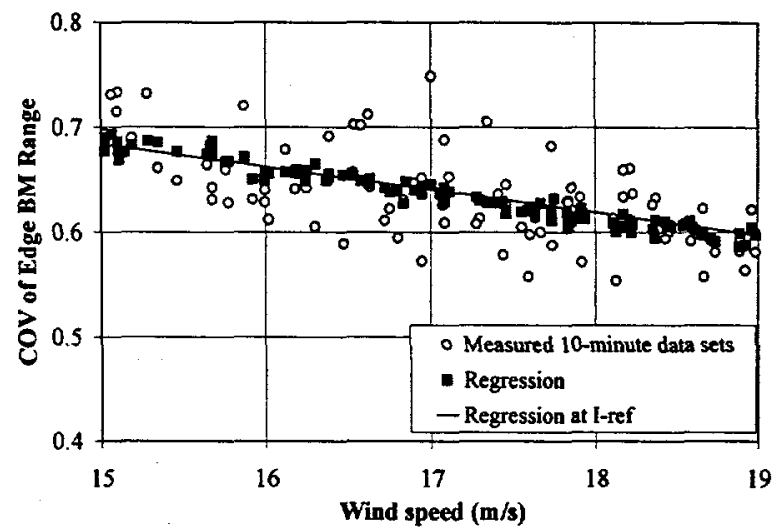

(b) Coefficient of variation (COV)

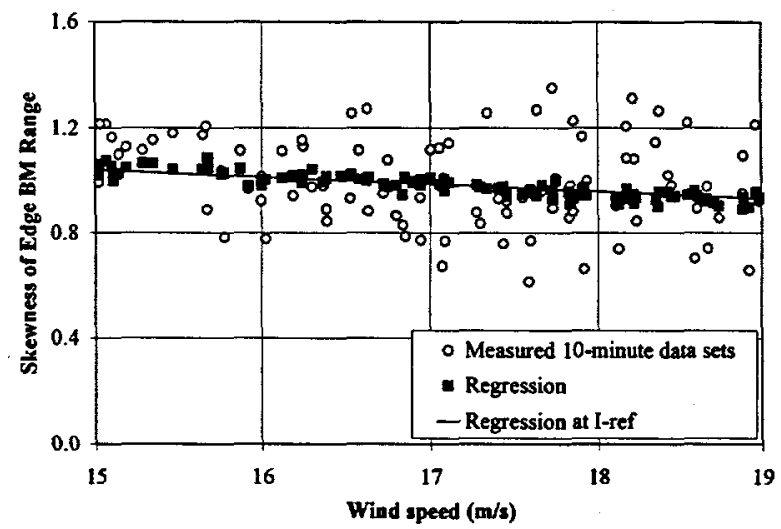

(c) Coefficient of skewness

Figure 7 Regression results of edge-wise bending moment range.

The Lavrio data set used in this example is limited to a range of wind speeds from 15 to $19 \mathrm{~m} / \mathrm{s}$. The long-term analysis in the next section will, for example purposes only, assume the regression trends found in high winds apply to all wind speeds. In an actual application, the data from a particular turbine will need to be examined over the entire range of damaging wind speeds. It might be amenable to regression fits that run all the way from cut-in to cut-out. More likely, the wind speed range will have to be partitioned into divisions over 
which the response moments are well behaved enough to be fit with simple regression. For example, it is likely that the response will have different characteristics above and below rated wind speed. In that case, the analysis presented here would have to be repeated for each wind-speed division before proceeding with the long-term analysis in the next section. It may also be the case that the response in low winds has an insignificant contribution to the fatigue damage and the analysis can safely deal with only high wind response. The individual application will determine the constraints.

\section{LONG-TERM ANALYSIS}

The long-term distribution of fatigue loads is obtained by integrating the short-term distributions (for loads conditional on wind conditions) over the specified distribution of wind conditions. Current IEC standards specify a Rayleigh distribution of wind speed with the annual average depending on class. Class I sites have a $10 \mathrm{~m} / \mathrm{s}$ average and Class II sites have a $8.5 \mathrm{~m} / \mathrm{s}$ average. Wind-speed classes defined as "Special" are also allowed with conditions that may be defined by the

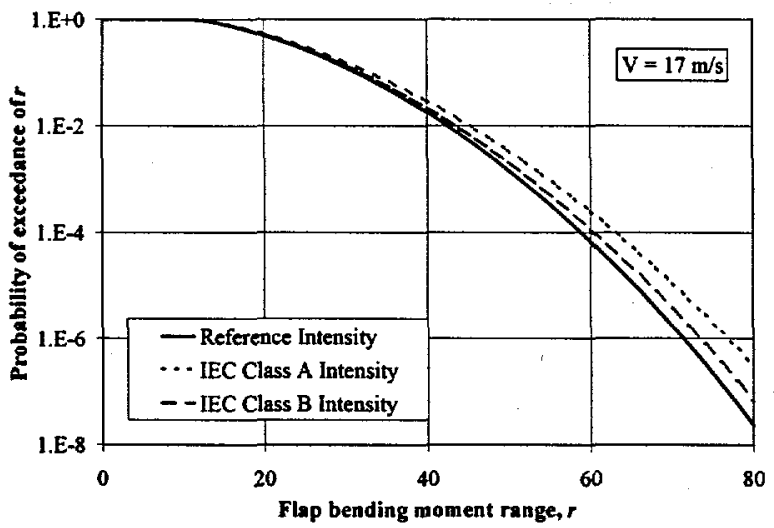

(a) Flap-wise bending

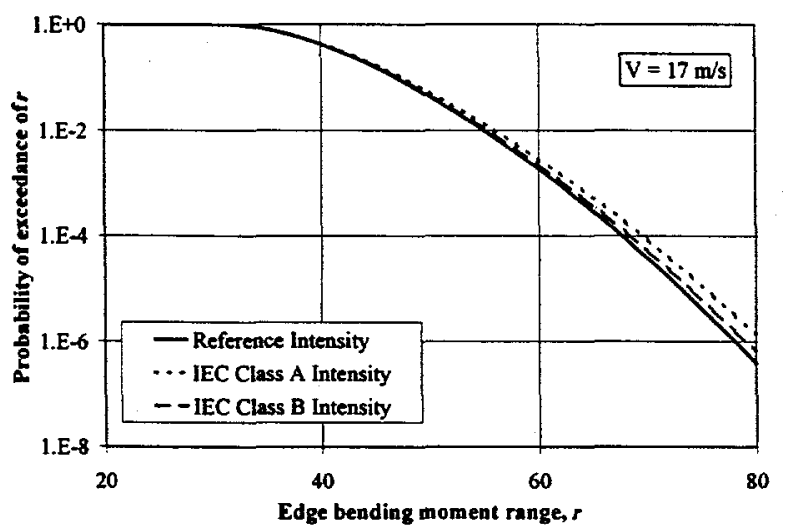

(b) Edge-wise bending

Figure 8 Distribution of bending moment ranges conditional on wind speed and turbulence intensity. designer. The turbulence intensity is a deterministic function of wind speed given by Eq. 1. A lifetime load distribution must sum all the short-term distributions at each wind speed and associated turbulence intensity weighting them by the annual wind speed distribution. This can be written as

$F(r)=\int F(r \mid V, I) f(V) d V$,

where $F(r)$ is the long-term distribution of stress ranges, $r$, and $F(r \mid V, I)$ is the short-term distribution of stress ranges conditional on the ten-minute average wind speed, $V$, and the specified turbulence intensity, $I . f(V)$ is the wind speed probability density function (PDF). The integration is carried out over all damaging wind speeds. The distribution functions of $r$ can be either the CDF or the exceedence (1-CDF). However, the integration must be over the probability density function for wind speed, $f(V)$.

Any environmental conditions can be used with Eq. 7 once the response moments have been defined with respect to the turbulence levels and wind speeds. This has been accomplished by the regression of the moments over $V$ and $I$ and by determining the shortterm distributions, $F(r \mid V, I)$, from the moments. As examples we will calculate the long-term distributions for two standard-driven and two site-driven environments.

Figures $9 \mathrm{a}$ and $9 \mathrm{~b}$ show the long-term distributions of flap and edge loads respectively for the IEC Class I wind speed distribution (Rayleigh $10 \mathrm{~m} / \mathrm{s}$ ) and for both turbulence Categories A and B. Both of these standard environments define the turbulence level as a function of wind speed by Eq. 1 .

The specification of a fixed turbulence intensity functionally related to the wind speed is somewhat artificial; measurements indicate that the turbulence intensity varies over a range of values for each tenminute sample (see Figure 1). A more realistic representation than Eq. 7 for the long-term distribution might be to include turbulence intensity as a random variable by integrating over both wind speed and turbulence intensity as follows:

$$
F(r)=\iint F(r \mid V, I) f(V, I) d I d V
$$

Figures $9 \mathrm{a}$ and $9 \mathrm{~b}$ show the long-term flap and edge distributions derived by integrating over both wind speed and turbulence assuming wind speed and turbulence variations are independent. (Independence implies that the joint PDF, $f V, I)$ in Eq. 8 is simply the product of the individual marginal PDFs, $f(V)$ and $f(I)$.) 


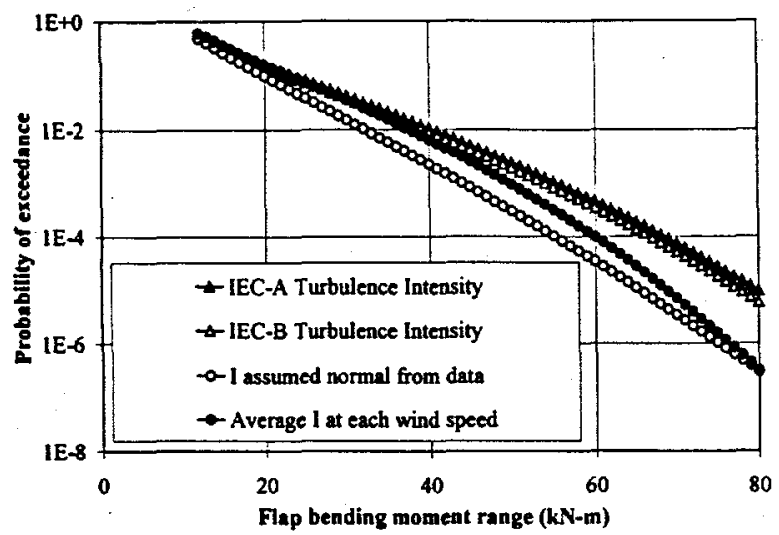

(a) Flap-wise bending

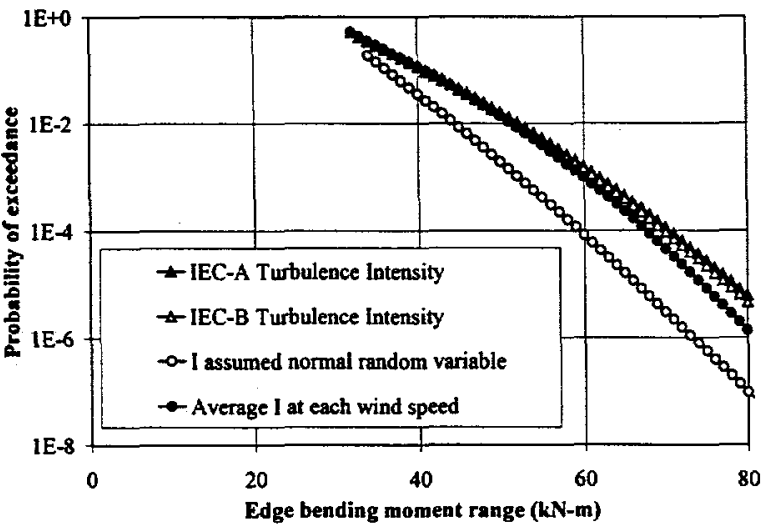

(b) Edge-wise bending

Figure 9 Long-term distribution of edge-wise bending moment ranges (Rayleigh distributed wind speed with mean $=10 \mathrm{~m} / \mathrm{s}$ ).

The wind speed is a $10 \mathrm{~m} / \mathrm{s}$-average Rayleigh distribution as prescribed for IEC Class I sites. The turbulence is assumed to be normally distributed with mean, $\bar{I}$, defined by $\bar{I} \cong 2.5 / V$, and standard deviation equal to 0.025 , based on a best fit to the data of turbulence vs. wind speed shown in Figure 10.

Also plotted in Fig. 9 is the result of assuming the turbulence at the Lavrio site is defined by the average value at each wind speed (the line with the black circles in Figure 10). This simpler assumption allows the use of Eq. 7. The comparison indicates that the integration over all turbulence levels, which is the most realistic reflection of the measurements, produces a much lower load spectrum. The simplified alternative, i.e., fixing the turbulence at the mean level, leads to a more conservative result, although the conservatism diminishes at very high load levels for the flap bending moment ranges. However, these results may be sensitive to the choice of a normal distribution for turbulence intensity.

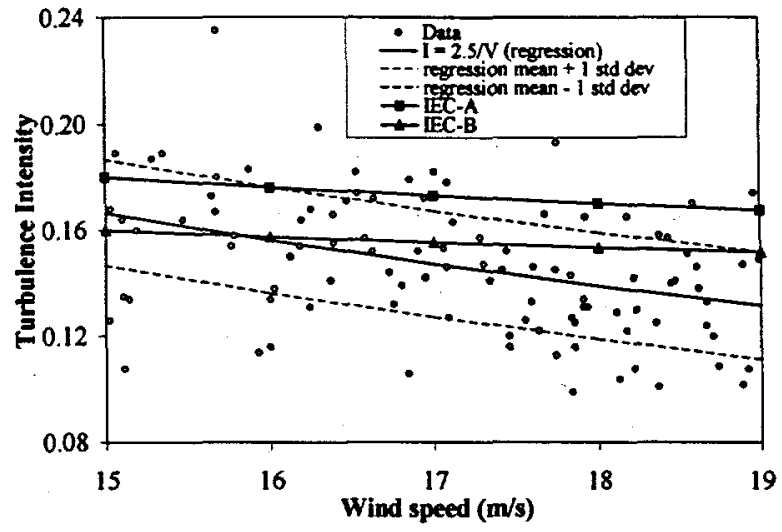

Figure 10 Lavrio site turbulence intensity as a function of wind speed, regression fits, and IEC Category $\mathrm{A}$ and $\mathrm{B}$ definitions.

\section{Discussion}

The Lavrio site's mean-plus-one-sigma turbulence intensity at $15 \mathrm{~m} / \mathrm{s}$ wind speed is quite similar to the IEC standard specification of $16 \%$ (Class B) to $18 \%$ (Class A). This similarity in turbulence levels is evident throughout the high wind range as shown in Figure 10. The differences between the distributions in Figures $9 \mathrm{a}$ and $9 \mathrm{~b}$ therefore provide an indication of the conservatism built into the IEC load cases relative to a fairly turbulent site.

Within the context of standards development, it may be reasonable to argue for lower turbulence specifications if differences as seen above can be shown to be significant and consistent. However, because the standards are based on past experience and industry consensus rather than objective risk-based analysis, it may be dangerous to remove conservatism from one area without also checking elsewhere to insure that this conservatism isn't covering for an unknown lack of conservatism elsewhere in the design process.

In general, the current standards give a load calculation "recipe" that is meant to meet some specific reliability criteria. If these current reliability levels are deemed adequate on average (over various cases), one cannot reduce conservatism in turbulence specification without adjusting the recipe to compensate elsewhere; e.g., through use of a higher load factor. Note that this alternative procedure - unbiased turbulence with higher load factor - may result in more uniform reliability across a range of cases. In contrast, current standards may lead to potential over-design of machines that are particularly sensitive to turbulence, and under-design in turbulence-insensitive cases. 


\section{ESTIMATING UNCERTAINTY IN LONG-TERM LOADS}

To review, the parametric load modeling proposed here proceeds by (1) modeling loads by their statistical moments $\mu_{i}(i=1,2,3)$ and (2) modeling each moment $\mu_{i}$ as a parametric function of $V$ and $I$ (Eq. 6). The moment-based model in step (1) is in principle independent of the turbine characteristics (although the optimal choice among such models may be somewhat case-dependent). Hence, in this parametric approach, the turbine characteristics are reflected solely through the moment relations in Eq. 6; specifically, the 9 coefficients $a_{i}, b_{i}, c_{i}(i=1,2,3)$. For clarity, we organize these here into a vector, $\boldsymbol{\theta}=\left\{a_{1}, b_{1}, c_{1}, a_{2}, b_{2}, c_{2}, a_{3}, b_{3}, c_{3}\right\}$.

Simpler 2-moment models would require only 6 coefficients.

The preceding section has shown one benefit of this parametric model. Because it permits load statistics to be estimated for arbitrary $V$ and $I$, the results can be weighted to form the long-term loads distribution as in Eqs. 7-8 (and Figs. 9a-b). Symbolically, we rewrite Eq. 7 here, noting explicitly its dependence on the vector $\theta$.

$$
F(r \mid \boldsymbol{\theta})=\int F(\boldsymbol{r} \mid V, I, \boldsymbol{\theta}) f(V) d V
$$

(Eq. 8 can be rewritten analogously.) The foregoing results (Figs 9a-b) have used our best estimates for the entries of $\theta$; i.e., the mean values of each entry in $\theta$. These are the values of $a_{i}, b_{i}$, and $c_{i}$ cited in Table 2 .

A further advantage of the parametric model lies in its usefulness in estimating the effects of statistical uncertainty. To clarify, it is useful to distinguish between the various terms in Eq. 9. The quantities $V$ and $I$ are "random variables;" that is, their future outcomes will show an intrinsic randomness that cannot be reduced by additional study of past wind conditions. In contrast, the 9 coefficients in $\boldsymbol{\theta}$ are in principle fixed (under the model's assumptions). We may, however, be uncertain as to their values due to limited response data. This "uncertainty" (as opposed to "randomness") can be reduced through additional sampling. The consequence of having only limited data can be reflected through $95 \%$ confidence levels, for example, on the exceedance probability $1-F(r)$. These are conceptually straightforward to establish by simulation. Assuming the entries of $\theta$ are each normally distributed, for example, one may (1) simulate multiple outcomes of $\theta$; (2) estimate $F(r)$ for each $\theta$ as in Eq. 9; and (3) sort the resulting $F(r)$ values (at each fixed $r$ value) to establish confidence bands; e.g., in which $95 \%$ of the values lie.

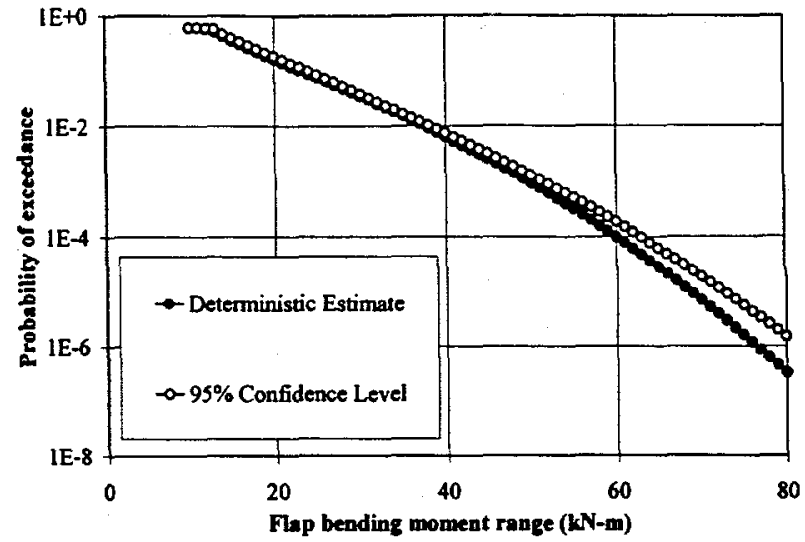

(a) Flap-wise banding

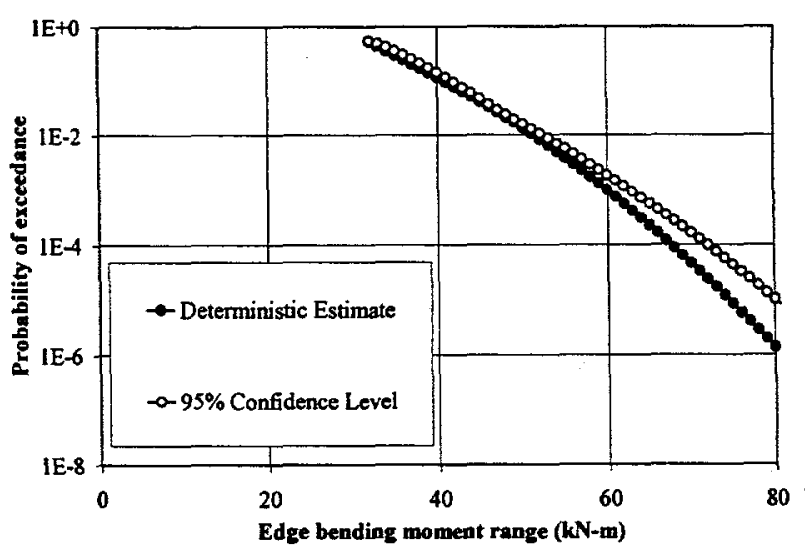

(b) Edge-wise Bending

Figure $1195 \%$ Confidence levels on the exceedance probability of fatigue loads for the Lavrio site with turbulence set to the average value for each wind speed.

Figures $11 \mathrm{a}$ and $11 \mathrm{~b}$ show the $95 \%$ confidence level on the exceedance probability, $1-F(r)$, which result from the simulation procedure described above. Each of the 9 coefficients in Eq. 9 were generated as statistically independent, normally distributed random variables, with means and standard deviations given by Tables 2 and 3 , respectively. (Correlation among these variables can also be included; however, this was not done here.) All of these results adopt the site-specific mean turbulence model; i.e., the results labeled "Average I at each wind speed" in Figs. 9a-b. These results from Figs. 9a-b are repeated in Figs. 11a-b, and referred to there as "deterministic" results. Also shown are $95 \%$ confidence results; i.e., probability levels below which $95 \%$ of the simulations fall.

The increase in probability, over the deterministic results in order to achieve $95 \%$ confidence, is found to be relatively modest. This reflects the benefit of having as many as 101 10-minute samples. If the same mean 
trends had resulted from fewer samples, the resulting $95 \%$ confidence results would be correspondingly higher than the mean results. Note also that, at least for flap-wise loads, the conservatism induced by the IEC turbulence models exceeds that required to cover our statistical loads uncertainty, based on the data at hand. Of course, as noted earlier, this IEC conservatism may be desirable to cover other sources of uncertainty. Finally, we caution again that these long-term load results are intended for example purposes only; accurate numerical values would require data across a broader range of wind speeds.

\section{SUMMARY}

Fatigue load spectra are generated for arbitrary site conditions (wind speed and turbulence intensity distributions) by using parametric models to fit the short term load spectrum to the first three moments of the truncated rainflow range distributions and regressing the moments over wind speed and turbulence intensity. The spectra are generated to specified IEC conditions for wind speed Class and turbulence Category. The spectra are also generated for asmeasured scatter in the turbulence levels across all wind speeds. The comparison of the two approaches reveals the level of conservatism that results from assumed high turbulence levels written into the current standards. The selected confidence level can be calculated using the statistics from regression analysis. Since the confidence interval depends on the uncertainty in the load characterization, it could provide a better margin of safety on the loads than can be accomplished with an inflated turbulence level. The parametric approach presented here illustrates how statistically based standards may be able to reflect the uncertainty in the loading definition caused by finitelength data records.

\section{ACKNOWLEDGMENTS}

The authors would like to extend a special debt of gratitude to the Centre for Renewable Energy Sources (CRES), Pikermi, Greece and especially to Fragiskos Mouzakis who found time in a busy schedule to supply the portion of the MOUNTURB data set used in the examples. The authors also gratefully acknowledge the efforts of LeRoy Fitzwater, of Stanford University, who provided the uncertainty analysis described in the final section of this paper.

\section{REFERENCES}

1. Fitzwater, L. M. and Winterstein, S. R., "Predicting Design Wind Turbine Loads from Limited Data: Comparing Random Process and Random Peak Models," A collection of the 2001 ASME Wind
Energy Symp., at the AIAA Aerospace Sciences Mtg., Reno, Nevada, AIAA-2001-0046, January 2001.

2. McCoy, T. J., Malcolm, D. J., and Griffin, D. A., "An Approach to the Development of Turbine Loads in Accordance with IEC 1400-1 and ISO 2394," A collection of the 1999 ASME Wind Energy Symp., at the AIAA Aerospace Sciences Mtg., Reno, Nevada, AIAA-99-0020, January 1999, pp. 1-9.

3. Ronold, K. O., Wedel-Heinen, J. and Christensen, C. J., "Reliability-based fatigue design of windturbine rotor blades," Elsevier, Engineering. Structures 21, 1999, pp. 1101-1114.

4. Veers, P. S. and Winterstein, S. R., "Application of Measured Loads to Wind Turbine Fatigue and Reliability Analysis," Journal of Solar Energy Engineering, Trans. of the ASME, Vol. 120, No. 4, November 1998.

5. IEC/TC88, 61400-1 Wind Turbine Generator Systems - Part 1: Safety Requirements, International Electrotechnical Commission (IEC), Geneva, Switzerland, 1998.

6. IEC/TC88, Draft IEC 61400-13 TS, Ed. 1: Wind turbine generator systems - Part 13: Measurement of mechanical loads, 88/120/CDV, International Electrotechnical Commission (IEC), Geneva, Switzerland, 1999.

7. MOUNTURB, Load and Power Measurement Program on Wind Turbines Operating in Complex Mountainous Regions, Volumes. I - III, Editor P. Chaviaropoulos, Coord. A. N. Fragoulis, CRES, RISO, ECN, NTUA-FS, published by CRES, Pikermi, Greece, Nov. 1996.

8. Butterfield, S., Holley, B., Madsen, P. H. and Stork, C., Report on $88 / 69 / C D$ - Wind Turbine Generator Systems Part 1: Safety Requirements, 2. Edition, National Renewable Energy Laboratory, Golden, CO, Oct. 1997.

9. Manuel, L., Kashef, T. and Winterstein, S. R., Moment-Based Probability Modelling and Extreme Response Estimation The Fits Routine, Version 1.2, SAND99-2985, Sandia National Laboratories, Albuquerque, NM, Nov. 1999.

10. Lange, C. H., Probabilistic Fatigue Methodology and Wind Turbine Reliability, SAND96-1246, Sandia National Laboratories, Albuquerque, NM, May 1996.

11. Nelson, D. V., and Fuchs, H. O., "Predictions of Cumulative Fatigue Damage using Condensed Load Histories," Fatigue under Complex Loading: Analysis and Experiments, Advances in Engineering, Vol. 6, Ed. R. M. Wetzel, SAE, Warrendale, PA, 1977, pp. 163-187. 